\title{
Solving Linear Systems of Equations using a Memetic Algorithm
}

\author{
Liviu Octavian Mafteiu-Scai \\ West University of Timisoara, \\ Timisoara, Romania
}

\author{
Emanuela Jana Mafteiu-Scai \\ National College „lancu de Hunedoara”, \\ Hunedoara, Romania
}

\begin{abstract}
This paper proposes a memetic algorithm (MA) to solve linear systems of equations, by transforming the linear system of equations into an optimization problem. Such exploitation of knowledge obtained in a local search/optimization allows the evolutionary programming implementation to produce very good results at a relatively low computational cost. The proposed MA is able to determine solutions of a given linear system of equations, even in cases where traditional methods fail ( determinant null, ill-conditioned systems, subdeterminate systems, supradeterminate systems, system doesn't satisfy the convergence conditions etc). In situations when a linear system of equations has multiple solutions, in proposed approach, the task is to find as many solutions as possible, inside of a given interval. In cases where no accurate solution for a linear system of equations exists, an approximate solution can be acceptable and it can be obtained by the proposed method.
\end{abstract}

\section{General Terms}

Algorithms.

\section{Keywords}

linear systems of equations, memetic algorithms

\section{INTRODUCTION}

\subsection{Solving systems of linear equations}

A frequent problem in numerical analysis is solving the linear systems of equations. That, consequently has generated a great interest among mathematicians and computer scientists, as evidenced by the large number of numerical methods developed over time. Besides the classical numerical methods, recently were proposed methods inspired by techniques from artificial intelligence. Hybrid methods have been also proposed along the time.

The classical methods are usually divided into exact/direct methods and iterative/indirect methods. In direct methods, the solution is obtained after a fixed number of operations, a number that is directly proportional to system size. In this case the solution is only affected by rounding errors and are preferred when the size of the system is acceptable. The most known direct methods are: Cramer, Gaussian elimination, Gauss-Jordan elimination, LU factorization, QR decomposition etc. In indirect methods the iterative process will be stopped after a preset number of steps or after fulfilling certain conditions. The process involves iterative appearance of truncation errors, the main disadvantage of these methods. However, because the rounding errors are not cumulated and because requiring a smaller number of operations, iterative methods are preferred especially for large systems of equations. The most known are: Gauss-Seidel method, Jacobi method, gradient and conjugate gradient methods, etc. The literature describing the classical methods is more than rich. As mentioned, for solving linear systems of equations there are known a lot of algorithms. But in the cases of general $n \times m$ matrices, when $n<m$ ( $m$ is the number of unknown variables and $n$ is the number of equations) the classical iterative algorithms are not applicable, with a few exceptions.

In the last years, techniques and methods from the field of artificial intelligence have been used to solve or preconditioning solving the systems of equations. In [1] is proposed an algorithm that uses a genetic approach to solve linear systems of equations. This problem is viewed in terms of an optimization task. There is defined a cost function with interval variables that is further minimized. The cost function used in the genetic algorithm is

$g=\max [a b s(f i)]$ for $i=1,2,3, \ldots, n$ where $\max [a b s(f i)]$ is the maximum absolute value of individual equations

$f i(x)=0$ of a system, with $n$ such equations. In [2], the authors investigate the applicability and effectiveness of genetic algorithms in finding the solutions of systems of linear equations. Solving a system of equations is regarded again as an optimization problem. The proposed algorithm is able to produce more than one set of solutions for a systems of equations. In that approach a chromosome is represented by a vector with integer values that corresponds to the unknowns of the system. The crossover and mutation operation are used in solving process. In terms of the objective function, the approach is similar to that in [1], but the problem is interpreted as a multiobjective optimization task. Such a multiobjective optimization approach is not new, since similar approaches were proposed also in $[3,4,5,7]$. In [6], the proposed method for solving a linear system of equations is based on using a particle swarm optimization algorithm and an artificial fish swarm algorithm, especially designed for illconditioned linear systems equations.

\subsection{Memetic algorithms and optimization problems}

The memetic algorithms (MAs) are inspired from genetic evolution and memetic/cultural evolution. Memetic algorithms are a family of metaheuristics for optimization that combine different algorithmic approaches for solving a problem. Memetic algorithms are in case of global optimization problems a good choice, that combine evolutionary techniques with other classical or intelligent optimization techniques. In most cases, MAs use a double optimization: local and global and represent a particular class of evolutionary algorithms that apply a local search in order to refine the current approximation of the global optimum. From another point of 
view, the MAs are population-based metaheuristics. A reference book that makes a detailed description of memetic algorithms is [8]. An extensive description of other natureinspired intelligent algorithms can be found in $[9,10,11,12]$.

The general structure of MA is presented in Algorithm 1

Algorithm 1:

Begin

Initialize Population

Repeat

Evaluate Curent Population

Select Parents

Crossover

Mutation

Evaluate New Chromosomes

Improve With Local Search

If Converged then Restart Population

Until TerminationCriterium

End

Some aspects are very important in terms of designing a good memetic algorithm:

the choice of recombination operators in the evolutionary process must take into consideration the three basic properties of these: purity, assortment and transmission [10];

a clear separation between local search/optimization and global search/optimization must be made. At the same time a balance between local and global search is required to avoid premature convergence and the waste of computing resources;

in case of population convergence, the population must be refreshed in order to avoid the exploration of the same search space, which would lead to obtaining the same solutions and unnecessary waste of processing time;

regarding the initial population, when is possible, is recommended a non-random initialization, which can direct the search into a particular regions that contain good or appropiate solutions. This can be achieved mainly by the inclusion in the initial population of good solutions previously known or by a process selected from a large population generated randomly (selection based on fitness);

the local search operators must be different from recombination and mutation operators of the evolutionary process;

using the knowledge acquired in previous optimization stages to guide the current phase of optimization is also an important aspect to be considered in designing a memetic algorithm.

\section{THE PROPOSED METHOD}

\subsection{The math problem}

Consider a system of $n$ linear equations with $n$ unknows that can be written in a vector equation form as:

$$
X_{1}\left[\begin{array}{c}
a_{11} \\
a_{21} \\
\cdot \\
\cdot \\
\cdot \\
a_{n 1}
\end{array}\right]+X_{2}\left[\begin{array}{c}
a_{12} \\
a_{22} \\
\cdot \\
\cdot \\
\cdot \\
a_{n 2}
\end{array}\right]+\cdots+X_{n}\left[\begin{array}{c}
a_{1 n} \\
a_{2 n} \\
\cdot \\
\cdot \\
\cdot \\
a_{n n}
\end{array}\right]=\left[\begin{array}{c}
b_{1} \\
b_{2} \\
\cdot \\
\cdot \\
\cdot \\
b_{n}
\end{array}\right]
$$

where, $x_{1}, x_{2}, \ldots . ., x_{n}$ are the unknowns, $a_{11}, a_{12}, \ldots . . a_{n n}$ are the coefficients of the system and $b_{1}, b_{2}, \ldots ., b_{n}$ are the constant terms.

Finding a solution for this linear system of equation $f(X)$ involves finding a solution such that every equation in the system is zero i.e.:

$$
\left\{\begin{array}{c}
f_{1}\left(x_{1}, x_{2}, \ldots, x_{n}\right)=0 \\
f_{2}\left(x_{1}, x_{2}, \ldots, x_{n}\right)=0 \\
\cdot \\
\cdot \\
f_{n}\left(x_{1}, x_{2}, \ldots, x_{n}\right)=0
\end{array}\right.
$$

where

$f_{i}\left(x_{i}\right)=a_{i 1} x_{1}+\cdots+a_{i n} x_{n}-b_{i}$

A solution is an assignment of values to the variables $x_{1}, x_{2}, \ldots, x_{n}$ such that each of the equation is satisfied. The solution set is the set of all possible solutions for the system. In case of a linear system of equations there are three possible situations:

single/unique solution $X$;

infinitely solutions $X$;

no solution.

In proposed approach, the problem of solving a linear system of equations is transformed into a multiobjective optimization problem, where each equation is used to define an objective function. The goal of these optimization functions is to minimize the difference between left side and right side for each equation in part, considered in absolute value.

From another point of view, the proposed method is a combined approach: an enumeration problem (finding a lot or all solution) and an optimization problem (finding a solution by minimized a given objective function). That function, named in term of evolutionary process, fitness function, tells the algorithm how good a particular solution is. The fitness functions chosen were $a b s\left(f_{i}(X)\right)$, for $i=1,2, \ldots, n$.

\subsection{General structure of proposed MA}

The general structure of the proposed MA for solving systems of linear equations is presented in Algorithm 2:

$\underline{\text { Algorithm2 }}$

Begin

Read linear system and other working parameters

Search Space Initialization

Generate Population in Search Space

repeat

Local Search and Optimization

Select Parents

Crossover 


\section{Mutation}

Global Search

If SolutionsFound then

If Solutions are New then

If Solutions are Approximate and Unsatisfactory then

RefiningSolutions

Output Solutions

Recorder Solutions

Expand Search Space

Generate Population in Search Space

until TerminationConditions

\section{End}

The algorithm for Generate Population in Search Space is presented below:

\section{Algorithm 3}

Begin

GenerateRandom Chromosomes in Search Space

Select PromisingChromosomes

Generate Non-RandomChromosomes

ReformingPopulation

If Degenerated then

Expand Search Space

Change Random_Generator for Generate Random

Chromosomes in Search Space

Generate Population in Search Space

\section{End}

As can be seen, Algorithm 3 is recursive when the population converges, but is a very low probability of an infinite loops because is used expanding the search space and changing the random generator.

\subsection{Basic elements of the proposed algoritm}

Chromosomes. A chromosome is a vector of $n$ integer/real values, corresponding to the unknowns of the system of equations. Should be mentioned that the case with real values and the case with integer values were treated separately when encoding the chromosomes.

Population. It consists of chromosomes that are particular solutions for each equation in part and chromosomes obtained by recombination and mutation, using the rules described in this paper.

Search Space. Because the search space is infinite $(-\infty,+\infty)$, it should be divided into intervals, which are successively investigated using the proposed MA. The issue was to decide the order of intervals investigation. In proposed approach, the start point is from the middle point $O$ with radius $r$, where $r$ is a real value set by the user. Then, in Expand Search Space process, the search intervals are increased successively to right and left with the same value $r$, preset by the user. For example, for $r=10$, the first search space is $[-10,10]$, the second will be $[-20,20]$ and so on. In contrast to other search processes in which the intervals are distinct, in our problem, such an approach would have been wrong because the components of the solution $X$ can be found in distinct intervals. The risk of a degenerate population and to rediscover local/global optimum has been removed by Expand Search Space and Change Random Generator.

Generate Random Chromosomes in Search Space. Random values are generated for the chromosomes of population. A chromosome in population is represented by a vector $S=\left\{x_{l}\right.$, $\left.x_{2} \ldots x_{n}\right\}$, with real values in the interval $[-r,+r]$. The $S$ vector corresponds to a set of values assigned to the system unknowns $X=\left\{x_{1}, x_{2} \ldots x_{n}\right\}$.

Select Promising Chromosomes. The chromosomes with the best fitness function values are selected, i.e. those which are closest to the system solution.

Generate Non-Random Chromosomes and Local Search and Optimization. For each equation (i), is considered its solution $X_{i}=\left\{x_{i l}, x_{i 2}, \ldots x_{i k} \ldots x_{i n}\right\}$. It is possible to calculate an accurate solution $X_{i}$ for each equation separately, starting from a randomly generated chromosome $S$. At first, it will replace the values of $S$ in equation $(i)$, and calculate the deviation from zero, in accordance to the fitness function. After, this deviation is distributed in proportion to each term of the equation (i). The $X_{i}$ values that are obtained representing a particular and accurate solution for the equation (i). Repeat the procedure for each equation separately. This procedure produces a set of individual and accurate solutions, one for each equation. The calculation procedure is described below.

Let $S$ be a randomly generated chromosome $S=\left\{s_{1}, s_{2}, \ldots\right.$ $\left.s_{k}, \ldots s_{n}\right\}$. An accurate value for $x_{i k}$, that is a component of $X_{i}=\left\{x_{i 1}, x_{i 2}, \ldots x_{i k} \ldots x_{i n}\right\}$, can be computed using:

$$
x_{i k}=s_{k}+d x_{i k}
$$

where $d x_{i k}$ represents the deviation of $s_{k}$ from the accurate value $x_{i k}$, and it is obtained using the formula:

$$
d x_{i k}=\frac{b_{i}-\sum_{j=1}^{n} a_{i j} * s_{j}}{n * a_{i k}}
$$

For example, for the following system:

$$
\left\{\begin{array}{c}
x+y+z=6 \\
x-y-z=-4 \\
-x-y+z=0
\end{array}\right.
$$

and a chromosome:

$$
S=\{3.0,-1.0,6.0\}
$$

the previously presented method produces:

$X_{1}=\{2.33,-1.66,5.33\}$ an accurate solution for eq. (1)

$X_{2}=\{2.33,-0.33,6.66\}$ an accurate solution for eq. (2)

$X_{3}=\{4.33,0.33,4.66\}$ an accurate solution for eq. (3)

After global search and evolutionary process is obtained the accurate solution of the linear system of equations example: $X_{1}=\{1,2,3\}, X_{2}=\{1,2,3\}$ and $X_{3}=\{1,2,3\}$. As mentioned above, $X_{i}=\left\{x_{1}, x_{2}, \ldots, x_{n}\right\}$ is the vector solution for equation $(i)$.

Global Search. In general, the ideal case is when the vectors $X_{i}$ are equal, and represents an accurate solution for the entire system of equations. If the vectors $X_{i}$ are not equal (the most common case), is computed the deviation for the entire system of equations $\operatorname{delta}(f(X))$ and the sum of deviations for each $x_{k}$ unknown in part, delta $\left(x_{k}\right)$. 


$$
\begin{gathered}
\operatorname{delta}(f(X))=\sum_{i=1}^{n}\left|b_{i}-\sum_{j=1}^{n} a_{i j} s_{j}\right| \\
\operatorname{delta}(X)=\sum_{k=1}^{n} \operatorname{delta}\left(x_{k}\right)=\sum_{k=1}^{n} \sum_{\substack{1 \leq i \leq n-1 \\
i+1 \leq j \leq n}}\left|x_{i k}-x_{j k}\right|
\end{gathered}
$$

The following indicators were introduced: $\operatorname{delta}(f(X))$ and $\operatorname{delta}\left(x_{i}\right)$. First, delta $(f(X))$, represents the sum of absolute deviations of each equation with accurate values $X_{i}$ from baseline $S$. Second, delta $\left(x_{k}\right)$, represents the sum of absolute deviation of each unknown $x_{i}$ calculated from $X_{i}$.The delta $(X)$ represent the sum of $\operatorname{delta}\left(x_{k}\right)$,

If these deviations fit into the limits set by the user, can be said that it was obtained an approximate solution for the system of equations. In practice, there are situations when such approximate solutions may be satisfactory from the user point of view. Maximum acceptable values for these deviations, noted $a_{a}$, initially set by the user, are part of the termination condition. At the same time, the deviation values obtained are considered, both for the global search process and for the evolutionaryprocess.

If the values obtained for $\operatorname{delta}(X)$ or $\operatorname{delta}(f(X))$ are zero, can say that there is an accurate solution for this system of equations.

The best values $X_{i}$ obtained at the moment $t$ will be used as values for $S$ at the moment $(t+1)$ in a process similar to that described in the previous section.

Expand Search Space. If the results obtained in the previous step are not satisfactory or the TerminationConditions are not satisfied, the search space $[-r,+r]$ will be expanded to right and left with the same value $r$, preset by the user.

Evolutionary process. In present approach a chromosome is represented by a vector $S=\left\{s_{1}, s_{2}, \ldots s_{n}\right\}$ with real values that corresponding to the unknowns of the system.

At the same time the best values obtained for $X_{i}$ in accordance with $\operatorname{delta}(X)$ and $\operatorname{delta}(f(X))$, will be used in the evolutionary process, in accordance with the principle of genetic elitism. In the evolutionary process, part of memetic algorithm, were applied to elite the following operations to create new chromosomes in the population: arithmetic average, geometric average, crossover and mutation, to ensure a high population diversity, but also to provide a search space close to the previoulsy obtained best values. The crossover process explores the search space around the already found good solutions. The mutation process explore the search space for new solutions.

In terms of number of crossovers performed, this is determined by the size of the system of equations. This value may be in the interval $\left[n, n^{2}\right]$, in case of middle point crossover, where $n$ is the size of the system.

The number of chromosomes obtained by mutation is also preset by the user. In the proposed method two types of mutations were performed. To ensure a high diversity of the population purely-random mutations were made in the search space $[-r,+r]$. To increase the likelihood of "promising" chromosomes, the mutations are based on small random variations of promising chromosomes, i.e. for small variations around the values of $X_{i}$.
To generate chromosomes by mutation, two generators were used for numbers, one for integers and another for real numbers. Integer values will be chosen in the vicinity of the best real values to speed up the convergence and save the computing resources.

In the initial population generation phase, is used a selective initialization that means a large number of random solutions are created and then the initial population is selected from these. Otherwise, two types of mutations were used. The first type is a purely-random mutation type, but situated only in the search space $[-r,+r]$, necessary to ensure a sufficient diversity and avoid premature convergence. The second type of mutation used, known as mass-mutation, consists of cloning the best chromosomes in order to produce a number of chromosomes in their vicinity. A non-random population based on elites can direct the search into a particular region of the search space that probably contain solution (best or closer solutions). The incorporation of the knowledge in the evolutionay cycle represents the essence of the memetic algorithm.

Degenerated populations. Once the population is considered to be at a degenerate state, the restarting population process must be invoked [8]. In the proposed method strategy is to keep a fraction of the current population (the current best chromosome) for the next population. The risk of a degenerate population and to rediscover local/global optimum has been removed by Expand Search Space and Change Random Function.

Optimization. Three criteria were used for optimization. The first criterion is applied locally for each equation separately and consists of minimizing the function $a b s\left(f_{i}(x)\right)=0$. The second criterion consists in determining/selecting $X_{i}$ vectors, for which $\operatorname{delta}\left(\left(f_{i}(x)\right)\right.$ are minimal. The third criterion is to determine/ select $X_{i}$ vectors for which $\operatorname{delta}\left(x_{i}\right)$ is minimal. The last two criteria are used in global optimization process, specifically in the selection of chromosomes which will contribute to the formation of new populations.

Termination Condition. The termination condition contains an iterator, initially set by the user, showing how many solutions are intended to be obtained, if there are infinite solutions. The minimum values for the $\operatorname{delta}\left(f_{i}(x)\right)$ and $\operatorname{delta}\left(x_{i}\right)$ are also used in the Termination Condition. If these values reach or fall below a certain value called admissible approximation $a_{a}$, the solution will be shown and the curent subprocess will be closed. The $a_{a}$ value is a real value in the interval $(0,1]$ and would be used to determine if an approximate solution for a linear system of equations is satisfactory for the user.

\section{EXPERIMENTAL RESULTS}

After the experiments, it was observed that the proposed method always finds a solution for a system of linear equations given, an accurates solution or an approximate solution, even in situations when conventional methods fail. This is in fact a confirmation of the results presented in $[1,2]$, the advantage of unconventional methods inspired by nature in comparison with conventional approaches. Such situations are exemplified in the examples 1, 2, 4 and 6, Table 1.

There are also situations when a linear system of equations has infinite solutions. The proposed MA algorithm can discover a great number of possible solutions in case of linear systems with infinite solutions. In such situations, conventional methods such as Gaussian eliminiation, Gauss- 
Jordan, Crammer, LU or Gauss-Seidel, determine a single solution of system or fail. To discover more than one solution (if exist) is used an incremental search of the initial interval. In present approach, the size of the incremental interval $r$ is set by the user. This advantage (finding multiple solutions) of using unconventional techniques, inspired by artificial intelligence, for solving systems of equations, is also mentioned in works such as [2, 3, 5]. In this paper, such a case is presented in Example 1, Table 1.

The proposed method is able to determine solutions of a system even if the number of equations is less than the number of unknowns, as can be seen in Example 7, Table 1. Also, the proposed method is able to determine solutions of a system even if the number of equations is larger than the number of unknowns, as can be seen in Example 8, Table 1.

In Table 1 are listed experimental solutions obtained for several systems of equations, using some traditional methods and the method proposed in this paper.

It should be mentioned that the results posted inTable 1, last column, obtained with the proposed MA are not refined with traditional algorithms. For example, refining the solutions obtained with the proposed MA in Example 3, Table 1, using Gaussian elimination, leads to the solution $\{-10.668,35.001$, $68.000\}$ which is much closer to the accurate solution.
Remark: It should be noted that mathematical software such as Mathematica can produce a set of linearly independent vectors from which linear system solutions can be constructed. In Table 2 are given solutions obtained with Mathematica for some equations systems from Table 1.

The values of the parameters used in the experiments are:

- $\quad r=10$

- $\quad$ population size of each generation $=1000$

- $\quad$ maxim number of generations $=100$

- $\quad$ maximum number of solutions inside an interval $=10$

- $\quad$ maximum acceptable deviations $a_{a}=10^{-10}$

It has been observed that the crossover technique does not help much, so the number of chromosomes generated by this technique was about $10 \%$ in a population.

As for mutations, it was observed that those that keep a part of the initial configuration of elites (mass-mutations) are the most productive.

Also, we observed that if we use a more random population, the method performance increases.

Table 1

\begin{tabular}{|c|c|c|c|c|c|c|c|c|c|}
\hline $\begin{array}{l}E \\
x\end{array}$ & System equations & Cramer & $\begin{array}{l}\text { Gauss- } \\
\text { eliminat } \\
\text { ion. }\end{array}$ & $\begin{array}{l}\text { Gauss- } \\
\text { Jordan }\end{array}$ & $\begin{array}{l}\text { LU } \\
\text { factorizati } \\
\text { on }\end{array}$ & $\begin{array}{l}\text { Gauss- } \\
\text { Seidel }\end{array}$ & $\begin{array}{l}\text { Genetic } \\
\text { Algorithm } \\
\text { [2] }\end{array}$ & $\begin{array}{l}\text { Conjugate gradient } \\
\text { (init.aprox.(0,0,0), } \\
\text { epsilon }=10^{-30} \text {, } \\
\text { max.iter. }=100)\end{array}$ & $\begin{array}{l}\text { Proposed } \\
\text { Memetic } \\
\text { Algorithm }\end{array}$ \\
\hline 1 & $\left\{\begin{array}{c}x+2 y+3 z=14 \\
x+y+z=6 \\
3 x+2 y+z=10\end{array}\right.$ & Fail & Fail & $\begin{array}{l}\{4, \\
-4, \\
6\}\end{array}$ & Fail & Fail & $\begin{array}{l}\{1,2,3\} \\
\{0,4,2\} \\
\{2,0,4\}\end{array}$ & $\begin{array}{l}\text { Fail } \\
\{4,-16,14\} \\
\text { Verify only first } \\
\text { equation }\end{array}$ & $\begin{array}{l}\{1,2,3\} \\
\{-2,8,0\} \\
\{0,4,2\} \\
\{2,0,4\} \\
\{4,-4,6\} \\
\{1.45,1.08, \\
3.45\} \\
\{-4.13,12.26,- \\
2.13\} \\
\{75.2,-146.3, \\
77.2\} \\
\{-493.7,991.3, \\
-491.6\}\end{array}$ \\
\hline 2 & $\left\{\begin{array}{c}x+y+z=6 \\
x-y-z=-4 \\
-x-y+z=0\end{array}\right.$ & $\begin{array}{l}\{1, \\
2, \\
3\}\end{array}$ & $\begin{array}{l}\{1, \\
2, \\
3\}\end{array}$ & $\begin{array}{l}\{1, \\
2, \\
3\}\end{array}$ & $\begin{array}{l}\{1, \\
2, \\
3\}\end{array}$ & Fail & $\mathrm{X}$ & $\begin{array}{l}\text { Fail } \\
\{1,3,2\} \\
\text { Verify only first and } \\
\text { second equation }\end{array}$ & $\{1,2,3\}$ \\
\hline 3 & $\left\{\begin{array}{c}-3 x+z=100 \\
2 y-z=2 \\
3 x+y=3\end{array}\right.$ & 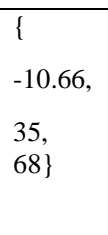 & $\begin{array}{l}\{ \\
-10.66, \\
35, \\
68\end{array}$ & $\begin{array}{l}\{ \\
-10.66, \\
35, \\
68\}\end{array}$ & $\begin{array}{l}\{-10.66, \\
35, \\
68\}\end{array}$ & $\begin{array}{l} \\
-10.66, \\
34.99, \\
68\}\end{array}$ & $\mathrm{X}$ & $\begin{array}{l}\text { Fail } \\
\{-108,-111, \\
-224\} \\
\text { Verify only first and } \\
\text { second equation }\end{array}$ & $\begin{array}{l}\{-10.58, \\
34.80 \\
68.27\}\end{array}$ \\
\hline 4 & & $\{1$, & $\{1$, & $\begin{array}{l}\{1.00, \\
2.00,\end{array}$ & $\begin{array}{l}\{0.99 \\
2.00\end{array}$ & Fail & $\{1,2,3\}$ & $\{1,2,3\}$ & $\{1,2,3\}$ \\
\hline
\end{tabular}




\begin{tabular}{|c|c|c|c|c|c|c|c|c|c|}
\hline & $\left\{\begin{array}{c}2 x+y+3 z=13 \\
x+5 y+z=14 \\
3 x+y+4 z=17\end{array}\right.$ & $\begin{array}{l}2, \\
3\}\end{array}$ & $\begin{array}{l}2, \\
3\}\end{array}$ & $2.99\}$ & $3.00\}$ & & & & \\
\hline 5 & $\left\{\begin{array}{c}2 x+4 y+z=5 \\
4 x+4 y+3 z=8 \\
4 x+8 y+z=9\end{array}\right.$ & $\begin{array}{l}\{0.5, \\
0.75 \\
1.0\}\end{array}$ & $\begin{array}{l}0.5 \\
0.75 \\
1.0\}\end{array}$ & $\begin{array}{l}\{0.50 \\
0.74 \\
0.99\}\end{array}$ & $\begin{array}{l}\{0.5, \\
0.75 \\
1.0\}\end{array}$ & $\begin{array}{l}\{0.50, \\
0.74 \\
0.99\}\end{array}$ & $\{0,1,1\}$ & $\begin{array}{l}\text { Fail } \\
\{-6.5,2.5,8\} \\
\text { Verify only first and } \\
\text { second equation }\end{array}$ & $\begin{array}{l}\{0.55 \\
0.72 \\
0.97\}\end{array}$ \\
\hline 6 & $\left\{\begin{array}{c}5 x+4 y-z=8 \\
x-y+z=4 \\
2 x+y+2 z=1\end{array}\right.$ & $\begin{array}{l}\{3.16, \\
-2.3 \\
-1.5\}\end{array}$ & $\begin{array}{l}\{3.16, \\
-2.33 \\
-1.50\}\end{array}$ & $\begin{array}{l}\{3.16 \\
-2.33 \\
-1.50\}\end{array}$ & $\begin{array}{l}\{3.16 \\
-2.33 \\
-1.50\}\end{array}$ & Fail & $\mathrm{X}$ & $\begin{array}{l}\text { Fail } \\
\{1.05,0.83,0.61\} \\
\text { Verify only first } \\
\text { equation }\end{array}$ & $\begin{array}{l}\{3.16, \\
-2.33 \\
1.49\}\end{array}$ \\
\hline 7 & $\left\{\begin{array}{c}5 x+4 y-z=8 \\
x-y+z=4\end{array}\right.$ & Fail & Fail & Fail & Fail & Fail & $\mathrm{X}$ & $\begin{array}{l}\text { Fail } \\
\{1,1,1\}\end{array}$ & $\begin{array}{l}\{1.59, \\
0.80, \\
3.20\}\end{array}$ \\
\hline 8 & $\left\{\begin{array}{c}x+y=2 \\
3 x-y=2 \\
-x+y=0\end{array}\right.$ & Fail & Fail & $\{1,1\}$ & Fail & Fail & $\mathrm{X}$ & $\begin{array}{l}\text { Fail } \\
\{2,0,0\} \\
\text { Verify only first } \\
\text { equation }\end{array}$ & $\{1,1\}$ \\
\hline 9 & $\left\{\begin{array}{c}10 x+y+z=12 \\
2 x+10 y+z=13 \\
2 x+2 y+10 z=14\end{array}\right.$ & $\begin{array}{l}\{1, \\
1, \\
1\}\end{array}$ & $\begin{array}{l}\{1, \\
1, \\
1\}\end{array}$ & $\begin{array}{l}\{1, \\
0.99,1\}\end{array}$ & $\begin{array}{l}1, \\
0.99 \\
1\}\end{array}$ & $\begin{array}{l}\{0.99, \\
1, \\
0.99\}\end{array}$ & $\{1,1,1\}$ & $\begin{array}{l}\{0.97 \\
1.08, \\
1.19\}\end{array}$ & $\{1,1,1\}$ \\
\hline $\begin{array}{l}1 \\
0\end{array}$ & $\left\{\begin{array}{c}x+2 y+3 z=6 \\
2 x+4 y+z=7 \\
3 x+2 y+9 z=14\end{array}\right.$ & $\begin{array}{l}\{1, \\
1, \\
1\}\end{array}$ & $\begin{array}{l}\{1, \\
1, \\
1\}\end{array}$ & $\begin{array}{l}\{0.99, \\
1, \\
1\}\end{array}$ & Fail & Fail & $\{1,1,1\}$ & $\begin{array}{l}\text { Fail } \\
\{1.40,0.79,1\} \\
\text { Verify only first and } \\
\text { second equation }\end{array}$ & $\{1,1,1\}$ \\
\hline $\begin{array}{l}1 \\
1\end{array}$ & $\left\{\begin{array}{c}2 x+4 y+8 z=44 \\
4 x+6 y+10 z=66 \\
6 x+8 y+10 z=84\end{array}\right.$ & $\begin{array}{l}\{4, \\
5, \\
2\}\end{array}$ & $\begin{array}{l}\{4, \\
5, \\
2\}\end{array}$ & $\begin{array}{l}\{4, \\
4.99,2\}\end{array}$ & $\begin{array}{l}\{4, \\
5, \\
2\}\end{array}$ & Fail & $\{4,5,2\}$ & $\begin{array}{l}\text { Fail } \\
\{13,-8.5,6.5\} \\
\text { Verify only first and } \\
\text { second equation }\end{array}$ & $\{4,5,2\}$ \\
\hline
\end{tabular}

$\mathrm{X}$ : results are not available for this case.

Table 2

\begin{tabular}{|c|c|c|c|}
\hline $\begin{array}{l}\text { Ex. no. } \\
\text { from } \\
\text { Table } 1\end{array}$ & System equations & Mathematica & $\begin{array}{l}\text { Proposed Memetic } \\
\text { Algorithm }\end{array}$ \\
\hline 1 & $\left\{\begin{array}{c}x+2 y+3 z=14 \\
x+y+z=6 \\
3 x+2 y+z=10\end{array}\right.$ & $\begin{array}{l}x=-2+z \\
y=8-2 z\end{array}$ & $\begin{array}{l}\{1,2,3\} \\
\{-2,8,0\} \\
\{0,4,2\} \\
\{2,0,4\} \\
\{4,-4,6\} \\
\{1.45,1.08,3.45\} \\
\{-4.13,12.26,-2.13\} \\
\{75.2,-146.3,77.2\} \\
\{-493.7,991.3,-491.6\}\end{array}$ \\
\hline 2 & $\left\{\begin{array}{c}x+y+z=6 \\
x-y-z=-4 \\
-x-y+z=0\end{array}\right.$ & $\begin{array}{l}x=1 \\
y=2 \\
z=3\end{array}$ & $\{1,2,3\}$ \\
\hline
\end{tabular}




\begin{tabular}{|l|l|l|l|}
\hline 3 & $\left\{\begin{array}{c}-3 x+z=100 \\
2 y-z=2 \\
3 x+y=3\end{array}\right.$ & $\mathrm{x}=-10.66$, & $\mathrm{y}=65.00$ \\
\hline 7 & $\left\{\begin{array}{c}5 x+4 y-z=8 \\
x-y+z=4\end{array}\right.$ & $\mathrm{y}=-1.33+0.66 \mathrm{z}$ & \\
\hline
\end{tabular}

\section{CONCLUSION AND FUTURE WORK}

It is concluded that MAs can be very helpful in finding solution for linear system of equations. Proposed MA worked accurate and were able to find solutions of the given linear system of equations, even in cases where traditional methods fail (determinant null, ill-conditioned systems, subdeterminate systems, supradeterminate systems, system doesn't satisfy the convergence conditions, convergence condition satisfied but an infinite number of solutions found/not found, etc). By this it can be concluded that the proposed MA is more effective than traditional numerical methods for solving linear systems of equations.

In cases where no accurate solution for a linear system of equations can not be determined, an approximate solution is good and it can be obtained by the proposed method. The proposed algorithm is able to discover solutions whose fitness is very close to 0 , which are good/acceptable in industrial applications.

There are situations when a linear system of equations has infinite solutions. In the present approach, the proposed method is able to find all the solutions inside of a given interval.

To discover more than one solution was used an incremental search of initial interval. In present approach, the size of incremental interval $r$ is set by the user. A further concern will be a dynamical size adjustment of the $r$ value, and even more, the determination of specific intervals and specific size of incremental interval $r$, different for each unknown in part.

The proposed method is relatively simple to implement, but it still requires some further implementation refinements. This is in particular for cases where the solutions obtained by the proposed method, "oscillates" around the accurate solution, but do not "catch" the accurate solution, as in the examples 3,5 and 6 , Table 1. While running proposed MA for the presented problems, it was observed that even if MA is able to search for all the system's solutions in a given interval $[-r$, $+r]$, in some cases, the MA accuracy is lower than the accuracy of solutions found by iterative methods. A solution is to use the proposed MA combined with traditional methods (gaussian, LU, GJ etc) for refining the solutions. That's because present approach is based on discrete optimization [8]. In this case, the solutions are characterized by their fitness values and can be accurate or in the vicinity of optimum. As a future direction of study, an approach to the problem as an continuous optimization [8] problem is recommended.

The MA schema developed above can also be implemented on parallel machines by allotting different equations to different machines in local search phase and this will be our preoccupation in the future.

\section{REFERENCES}

[1] Al Dahoud Ali , Ibrahiem M. M. El Emary, and Mona M. Abd El-Kareem, Application of Genetic Algorithm in Solving Linear Equation Systems, MASAUM Journal of Basic and Applied Science, Vol.1, No.2 Sept. 2009

[2] Ikotun Abiodun M., Lawal Olawale N.,Adelokun Adebowale P. The Effectiveness of Genetic Algorithm in Solving Simultaneous Equations, International Journal of Computer Applications (0975 - 8887) Volume 14- No.8, February 2011

[3] Crina Grosan, Ajith Abraham, Multiple Solutions for a System of Nonlinear Equations, International Journal of Innovative Computing, Information and Control ICIC International , 2008 ISSN 1349-4198

[4] Ibrahiem M.M. El-Emary,Mona M. Abd El-Kareem, Towards Using Genetic Algorithm for Solving Nonlinear Equation Systems, World Applied Sciences Journal 5 (3): pp. 282-289, 2008, ISSN 1818-4952

[5] Crina Grosan, Ajith Abraham, A New Approach for Solving Nonlinear Equations Systems, IEEE Transaction on Systems, Man and Cybernetics-part A: Systems and Humans, vol. 38, no. 3, May 2008

[6] Yong Zhou, Huajuan Huang, Junli Zhang, Hybrid Artificial Fish Swarm algorithm for Solving IllConditioned Linear Systems of Equations, ICICIS 2011 Proceedings, Part 1, Springer 2011, pp. 656-662

[7] Nikos E. Mastorakis, Solving Non-linear Equations via Genetic Algorithms, Proceedings of the 6th WSEAS Int. Conf. on Evolutionary Computing, Lisbon, Portugal, June 16-18, 2005 pp24-28

[8] Ferrante Neri, Carlos Cotta, and Pablo Moscato (Eds.), Handbook of Memetic Algorithms, Studies in Computational Intelligence, 2012 Springer-Verlag Berlin Heidelberg, ISBN 978-3-642-23246-6

[9] Jason Brownlee, Clever Algorithms: Nature-Inspired Programming Recipes, 2011, ISBN:978-1-4467-8506-5

[10] Michel Gendreanu, J.Y.Potvin, Handbook of Metaheuristics, Springer 2010, ISBN 978-1-4419-1663-1

[11] Teofilo F. Gonzales, Handbook of Approximation Algorithms and Metaheuristics, Chapman\&Hall/CRC 2007

[12] N. Krasnogor, Studies in the Theory and Design Space of Memetic Algorithms, PhD thesis, University of the West of England, 2002 